\title{
Lithium abundance in lower red giant branch stars of Omega Centauri ${ }^{\star} \star \star \star$
}

\author{
A. Mucciarelli ${ }^{1,2}$, M. Salaris ${ }^{3}$, L. Monaco ${ }^{4}$, P. Bonifacio $^{5}, \mathrm{X} . \mathrm{Fu}^{1,2}$, and S. Villanova ${ }^{6}$ \\ 1 Dipartimento di Fisica e Astrono mia, Università degli Studi di Bologna, Via Gobetti 93/2, 40129 Bologna, Italy \\ e-mail: alessio.mucciarelli2@unibo.it \\ 2 INAF - Osservatorio di Astrofisica e Scienza dello Spaziodi Bologna, Via Gobetti 93/3, 40129 Bologna, Italy \\ 3 Astrophysics Research Institute, Liverpool John Moores University, 146 Brownlow Hill, L3 5RF Liverpool, UK \\ ${ }_{5}$ Departamento de Ciencias Fisicas, Universidad Andres Bello, Fernandez Concha 700, Las Condes, Santiago, Chile \\ 5 GEPI, Observatoire de Paris, Université PSL, CNRS, Place Jule Janssen, 92190 Meudon, France \\ ${ }^{6}$ Universidad de Concepción, Casilla 160-C, Concepción, Chile
}

Received 20 May 2018 / Accepted 19 July 2018

\begin{abstract}
We present $\mathrm{Li}, \mathrm{Na}, \mathrm{Al}$, and $\mathrm{Fe}$ abundances of 199 lower red giant branch star members of the stellar system Omega Centauri, using high-resolution spectra acquired with FLAMES at the Very Large Telescope. The $A(\mathrm{Li})$ distribution is peaked at $A(\mathrm{Li}) \sim 1 \mathrm{dex}$ with a prominent tail towards lower values. The peak of the distribution well agrees with the lithium abundances measured in lower red giant branch stars in globular clusters and Galactic field stars. Stars with $A(\mathrm{Li}) \sim 1$ dex are found at metallicities lower than $[\mathrm{Fe} / \mathrm{H}] \sim-1.3$ dex but they disappear at higher metallicities. On the other hand, Li-poor stars are found at all metallicities. The most metal-poor stars exhibit a clear $\mathrm{Li}-\mathrm{Na}$ anti-correlation, where about $30 \%$ of the sample have $A(\mathrm{Li})$ lower than $\sim 0.8$ dex, while these stars represent a small fraction of normal globular clusters. Most of the stars with $[\mathrm{Fe} / \mathrm{H}]>-1.6 \mathrm{dex}$ are Li poor and Na rich. The Li depletion measured in these stars is not observed in globular clusters with similar metallicities and we demonstrate that it is not caused by the proposed helium enhancements and/or young ages. Hence, these stars formed from a gas already depleted in lithium. Finally, we note that Omega Centauri includes all the populations (Li-normal/Na-normal, Li-normal/Na-rich, and Li-poor/Na-rich stars) observed, to a lesser extent, in mono-metallic GCs.
\end{abstract}

Key words. stars: abundances - stars: atmospheres - stars: Population II - globular clusters: individual: Omega Centauri stars: evolution

\section{Introduction}

The emergent and generally accepted picture of stellar populations in globular clusters (GCs) is that these stellar systems host various (chemically distinct) stellar populations. In the majority of the cases, the stars of a GC share the same abundances of most of the elements, in particular Fe and iron-peak elements (see e.g. Carretta et al. 2009c; Willman \& Strader 2012). This demonstrate that these systems are not able to retain the ejecta of the supernovae. On the other hand, light elements $(\mathrm{C}, \mathrm{N}, \mathrm{O}, \mathrm{Na}, \mathrm{Mg}$, and $\mathrm{Al}$ ) exhibit large star-to-star variations and often coherent patterns, i.e. $\mathrm{C}-\mathrm{N}, \mathrm{Na}-\mathrm{O}$, and $\mathrm{Mg}-\mathrm{Al}$ anti-correlations (see e.g. Smith \& Norris 1982; Kraft et al. 1992; Carretta et al. 2009a,b; Gratton et al. 2012; Meszaros et al. 2015; Pancino et al. 2017; Bastian \& Lardo 2018). These chemical patterns are usually interpreted as the signature of internal pollution by low-energy ejecta of stars where high-temperature proton-capture cycles (CNO, NeNa, and $\mathrm{MgAl}$ cycles) have occurred. The so-called first population (1P) of the cluster is composed by stars with the same chemical composition of the original cloud from which the cluster formed. Instead, the subsequent second populations (2P) stars show variations of the light element abundances and they

\footnotetext{
$\star$ Based on observations collected at the ESO-VLT under programme 096.D-0728

$\star \star$ Full Tables 1 and 2 are only available at the CDS via anonymous ftp to cdsarc.u-strasbg.fr (130.79.128.5) or via http://cdsarc.u-strasbg.fr/viz-bin/qcat?J/A+A/618/A134
}

are expected to form from the pristine gas diluted with the ejecta of the polluter stars. A complete and quantitatively successful model for this self-enrichment process is still lacking because there is still debate about several details of the model (see e.g. Renzini et al. 2015; Bastian \& Lardo 2015), for instance the identification of the main polluter stars. The most favorite candidate polluter stars are asymptotic giant branch (AGB) stars with masses of 4-8 $M_{\odot}$ (Ventura et al. 2001; D'Ercole et al. 2008, 2010) and fast rotating massive stars (Meynet et al. 2006; Prantzos \& Charbonnel 2006; Decressin et al. 2007).

The abundance of lithium, $A(\mathrm{Li})^{1}$, in GC stars is a valuable diagnostic to understand this self-enrichment process, which poses challenges to the current theoretical models for the formation/evolution of GCs. Lithium is one of the few elements created during the Big Bang nucleosynthesis, together with $\mathrm{H}$ and $\mathrm{He}$. In the subsequent evolution of the stars, $\mathrm{Li}$ is destroyed in the stellar interiors owing to proton capture reactions occurring at $\sim 2.5 \times 10^{6} \mathrm{~K}$. However, Li should be preserved in the stellar envelopes of unevolved stars or partially diluted in the photospheres of stars that have undergone processes of mixing. The main mixing episodes are the first dredge-up (FDU) after the completion of the main sequence stage, and the extra-mixing episode occurring at the luminosity level of the red giant branch (RGB) bump. Because the proton-capture reactions responsible for the chemical anomalies observed in GC stars occurred at tempera-

\footnotetext{
$1 A(\mathrm{Li})=\log \frac{n(\mathrm{Li})}{n(\mathrm{H})}+12.00$.
} 
tures significantly higher $\left(>10^{7} \mathrm{~K}\right)$ than that of the Li burning $\left(\sim 10^{6} \mathrm{~K}\right)$, the enriched material from which the $2 \mathrm{P}$ stars formed should be $\mathrm{Li}$ free. Therefore, $\mathrm{a} \mathrm{Li}-\mathrm{Na}$ anti-correlation and a $\mathrm{Li}-\mathrm{O}$ correlation are expected within the individual clusters.

Abundances of Li in GCs have been obtained from dwarf and lower RGB (LRGB) stars. The latter are stars located between the FDU and RGB bump. Even if measures of $A(\mathrm{Li})$ are limited to a few GCs, the emerging scenario turns out to be complex and some GCs show evidence of star-to-star scatter in $A(\mathrm{Li})$, such as in the cases of NGC 6397 (Lind et al. 2009; Gonzalez Hernandez et al. 2009), NGC 6752 (Pasquini et al. 2005; Shen et al. 2010), M4 (Monaco et al. 2012), NGC 2808 (D’Orazi et al. 2015), and 47 Tucanae (D'Orazi et al. 2010; Dobrovolskas et al. 2014). Other clusters (M92, NGC 362, NGC 1904, NGC5904, and NGC 6218; Bonifacio 2002; D'Orazi et al. 2014, 2015) show a remarkably homogeneous $\mathrm{Li}$ abundance. Up to now the only clusters that exhibit undeniable evidence of correlations between Li and light elements are NGC 6752, which shows both a Li-O correlation (Shen et al. 2010) and a Li-Na anti-correlation (Pasquini et al. 2005); NGC 6397, where three Li-poor, Na-rich dwarf stars have been found (Lind et al. 2009); and NGC 2808, where some Al-rich RGB stars have $A(\mathrm{Li})$ lower than that in other stars with similar Al content (D'Orazi et al. 2015). In M4 a hint of Li-Na anti-correlation is found (Monaco et al. 2012) and has a larger $A(\mathrm{Li})$ scatter among $2 \mathrm{P}$ stars with respect to $1 \mathrm{P}$ stars but no evidence of Li-O correlation (Mucciarelli et al. 2011). 47 Tucanae shows the opposite situation; there is a statistically significant Li-O correlation, but no clear evidence of a Li-Na anti-correlation (Dobrovolskas et al. 2014).

In this paper we investigate the $\mathrm{Li}$ content in the stellar system Omega Centauri (NGC 5139) using LRGB stars and we aim to highlight possible correlations with abundances of light elements involved in the chemical anomalies. Traditionally classified as a GC, Omega Centauri exhibits (at variance with the other globulars) a wide iron distribution (Johnson et al. 2008, 2009; Johnson \& Pilachowski 2010; Villanova et al. 2014; Pancino et al. 2011a; Marino et al. 2011) and a variety of discrete sub-sequences in its colour-magnitude diagram (CMD, Lee et al. 1999; Pancino et al. 2000; Ferraro et al. 2004; Bedin et al. 2004; Sollima et al. 2005a, 2007; Milone et al. 2008), suggesting that this system has been able to retain the ejecta of the supernovae, experiencing a chemical enrichment history more complex than that of a normal GC. According to this evidence, Omega Centauri is usually interpreted as the remnant of a disrupted dwarf spheroidal galaxy (Bekki \& Freeman 2003). On the other hand, its dominant (metal-poor) population shows a clear $\mathrm{Na}-\mathrm{O}$ anticorrelation (Johnson \& Pilachowski 2010; Marino et al. 2011), demonstrating that the same self-enrichment process observed in mono-metallic GCs also occurred in this stellar system.

The only determination of $A(\mathrm{Li})$ in Omega Centauri stars has been presented by Monaco et al. (2010) who analysed a sample of 91 dwarf stars in the iron range $[\mathrm{Fe} / \mathrm{H}]=-2.0 /-1.4 \mathrm{dex}$, finding an average lithium $A(\mathrm{Li})=2.19 \mathrm{dex}(\sigma=0.14 \mathrm{dex})$. This abundance is compatible with the values usually measured among the Galactic dwarf stars. Measures for light element abundances are not available for this sample of dwarf stars. Because of the faintness of the dwarf stars in Omega Centauri $(V>18)$, large samples of high quality spectra can only be obtained with a huge amount of telescope time. The observation of LRGB stars (instead of dwarf stars) allows us to study the lithium content in Omega Centauri using a large sample of high signal-to-noise $(\mathrm{S} / \mathrm{N})$, high-resolution spectra, coupling these abundances with those of the light elements to study the lithium content in $1 \mathrm{P}$ and $2 \mathrm{P}$ stars.

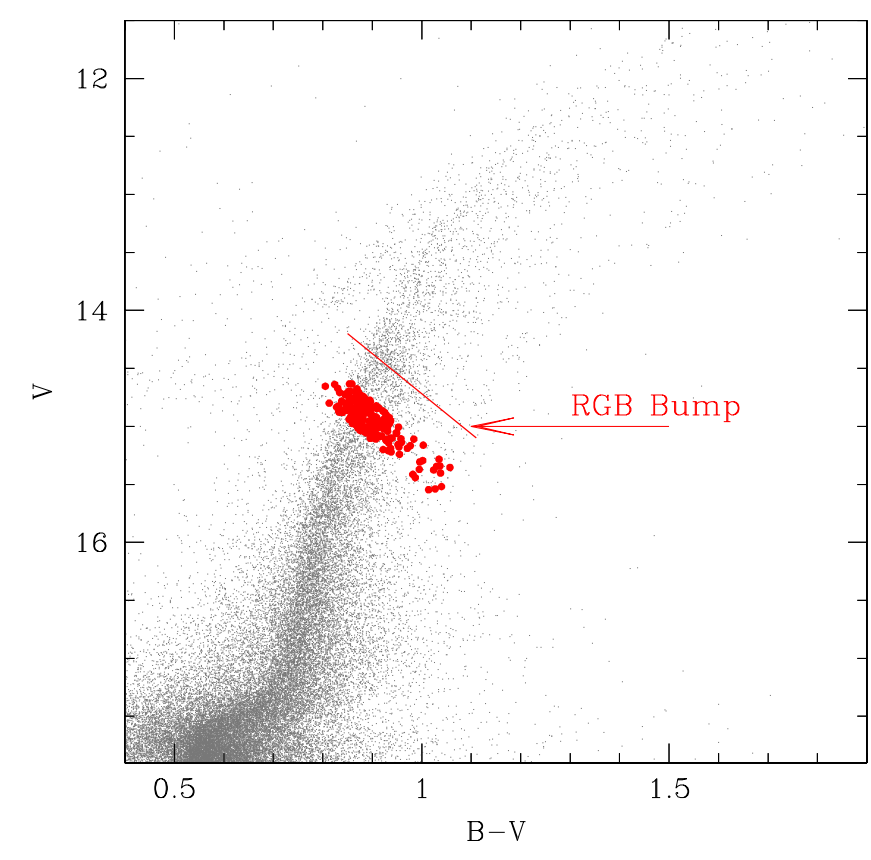

Fig. 1. Colour-magnitude diagram of Omega Centauri (Bellini et al. 2009 , stars selected within 750 arcsec from the cluster centre). The position of the spectroscopic targets (red circles) and the mean locus (red line) of the RGB bump of the various stellar populations of Omega Centauri are shown.

The paper is structured as follows: Sect. 2 describes the observations and selection of the member stars of Omega Centauri; Sect. 3 describes the adopted procedure for the chemical analysis; and the results are presented in Sects. 4-6 and discussed in Sect. 7.

\section{Observations and membership}

The observations have been collected under the ESO programme 096.D-0728 (PI: Mucciarelli) using the multi-object spectrograph FLAMES (Pasquini et al. 2000) at the Very Large Telescope. The GIRAFFE+UVES combined mode was used, allowing the simultaneous allocation of 132 mid-resolution GIRAFFE fibres and 8 high-resolution UVES (Dekker et al. 2000) fibres.

All the stars were observed with three FLAMES-GIRAFFE set-ups, namely HR12 $(\Delta \lambda=5821-6146 \AA, R \sim 20000)$, $\operatorname{HR} 13(\Delta \lambda=6120-6405 \AA, R \sim 26000)$, and HR15N $(\Delta \lambda=$ 6470-6790 $\AA, R \sim 19000)$, allowing the measure of several Fe spectral lines, as well as the Li line at $6708 \AA$, the Na D doublet at 5890-5896 $\AA$, and the Al I doublet at 6696-6698 $\AA$. Two observations of $1350 \mathrm{~s}$ each have been secured for HR12: four exposures of $1800 \mathrm{~s}$ each for HR12 and six exposures of $2700 \mathrm{~s}$ each for HR15N. Two target configurations have been taken, observing a total of 211 stars.

The targets were selected from the Bellini et al. (2009) BVRI WFI $2.2 \mathrm{~m}$ photometric catalogue; stars $\sim 0.6 \mathrm{mag}$ fainter than the mean locus of the RGB bump were picked. These stars are hence located after the completion of the FDU and before the onset of the extra mixing usually observed after the RGB bump. Figure 1 shows the positions of the observed targets (red circles), together with the mean locus of the RGB bump (red thick line). Only stars without close companions of comparable or brighter luminosity were selected to avoid stellar contamination within the fibres. 
Table 1. Main information on the member LRGB targets of Omega Centauri.

\begin{tabular}{cccccccc}
\hline \hline ID & $\begin{array}{c}\text { RA } \\
(J 2000)\end{array}$ & $\begin{array}{c}\text { Dec } \\
(\mathrm{J} 2000)\end{array}$ & $B$ & $V$ & $I$ & $K_{\mathrm{s}}$ & $\begin{array}{c}\mathrm{RV}_{\text {hel }} \\
\left(\mathrm{km} \mathrm{s}^{-1}\right)\end{array}$ \\
\hline 22580 & 201.723137 & -47.661730 & 15.630 & 14.747 & 13.660 & 12.293 & $+216.2 \pm 0.2$ \\
25939 & 201.832739 & -47.653890 & 15.818 & 14.950 & 13.874 & 12.394 & $+227.8 \pm 0.1$ \\
31135 & 201.784629 & -47.643055 & 15.707 & 14.843 & 13.760 & 12.370 & $+239.0 \pm 0.1$ \\
35536 & 201.750357 & -47.634749 & 15.634 & 14.763 & 13.677 & 12.269 & $+224.8 \pm 0.1$ \\
45849 & 201.765455 & -47.617715 & 15.616 & 14.762 & 13.752 & 12.461 & $+252.3 \pm 0.1$ \\
49844 & 201.735347 & -47.611785 & 15.890 & 14.981 & 13.868 & 12.450 & $+223.9 \pm 0.1$ \\
56555 & 201.683982 & -47.602406 & 16.002 & 15.053 & 13.948 & 12.548 & $+218.5 \pm 0.1$ \\
57780 & 201.709221 & -47.600836 & 15.972 & 15.041 & 13.951 & 12.592 & $+224.0 \pm 0.2$ \\
64366 & 201.683249 & -47.592755 & 15.904 & 15.013 & 13.914 & 12.460 & $+224.6 \pm 0.2$ \\
67162 & 201.724149 & -47.589388 & 15.880 & 14.976 & 13.897 & 12.504 & $+245.2 \pm 0.1$ \\
70485 & 201.812966 & -47.585439 & 15.568 & 14.723 & 13.644 & 12.303 & $+237.4 \pm 0.1$ \\
71476 & 201.601550 & -47.584175 & 15.551 & 14.699 & 13.641 & 12.246 & $+227.1 \pm 0.2$ \\
73028 & 201.820008 & -47.582544 & 15.665 & 14.787 & 13.706 & 12.339 & $+233.3 \pm 0.1$ \\
73743 & 201.920362 & -47.581721 & 15.851 & 14.985 & 13.907 & 12.533 & $+253.1 \pm 0.1$ \\
73986 & 201.741336 & -47.581473 & 15.683 & 14.787 & 13.692 & 12.197 & $+259.6 \pm 0.1$ \\
74878 & 201.892105 & -47.580466 & 15.712 & 14.846 & 13.764 & 12.419 & $+243.7 \pm 0.1$ \\
77093 & 201.563334 & -47.578011 & 15.792 & 14.919 & 13.832 & 12.487 & $+222.1 \pm 0.2$ \\
\hline
\end{tabular}

Notes. Coordinates and $B, V, I$ magnitudes are from Bellini et al. (2009), $K_{\mathrm{s}}$ magnitudes are 2MASS database (Skrutskie et al. 2006). This table is available in its entirety at the CDS.

All the spectra were reduced with the standard ESO GIRAFFE pipeline ${ }^{2}$, including bias-subtraction, flat-fielding, wavelength calibration with a reference Th-Ar lamp, and extraction of the one-dimensional spectra.

Radial velocities (RVs) were measured for each individual spectrum from the position of several metallic lines using the code DAOSPEC (Stetson \& Pancino 2008). After the correction for the corresponding heliocentric velocity, the spectra of each star were coadded together and used for the chemical analysis. Typical S/N per pixel are 70-100 for HR12, 120-150 for HR13, and $180-230$ for HR15N.

We excluded from the following chemical analysis six stars with RV not compatible with that of Omega Centauri and four stars with RV dispersion larger than $1.7 \mathrm{~km} \mathrm{~s}^{-1}$ (while all the other targets have RV dispersions significantly smaller than $1 \mathrm{~km} \mathrm{~s}^{-1}$ ) and considered as candidate binary stars. Finally, we derived chemical abundances for a total of 201 member stars of Omega Centauri. All the main information (coordinates, magnitudes, and RVs) of the member stars are listed in Table 1.

\section{Chemical analysis}

Effective temperatures $\left(T_{\text {eff }}\right)$ and surface gravities $(\log g)$ were derived from the photometric information. The values $T_{\text {eff }}$ were obtained by averaging $T_{\text {eff }}$ derived from $(B-V)_{0},(V-I)_{0}$ and $\left(V-K_{\mathrm{s}}\right)_{0}$ from the Alonso et al. (1999) transformations. The $B$, $V$, and $I$ magnitudes are from Bellini et al. (2009), $K_{\mathrm{s}}$ magnitude from 2MASS database (Skrutskie et al. 2006). Because the Alonso et al. (1999) calibration adopted the Johnson I-filter, the Cousin I-band magnitudes by Bellini et al. (2009) were transformed to the Johnson photometric system adopting the transformation provided by Bessell (1979). The $K_{\mathrm{s}} 2 \mathrm{MASS}$ magnitudes were transformed to the Telescopio Carlos Sanchez photometric system (used by Alonso et al. 1999) by means of the relations by Carpenter (2001) and Alonso et al. (1998).

\footnotetext{
2 http://www.eso.org/sci/software/pipelines/
}

The dereddened colours were obtained by adopting the extinction coefficients from McCall (2004) and the colour excess quoted in the Harris (1996) catalogue $(E(B-V)=0.12 \mathrm{mag})$. We note that Omega Centauri is not significantly affected by differential reddening (Bellini et al. 2009) and this effect was neglected in the atmospheric parameters determination,

2MASS $K_{\mathrm{s}}$ magnitudes are available for 143 stars and, for these, we averaged the three $T_{\text {eff }}$ values. For 54 stars we used only $T_{\text {eff }}$ derived from $(B-V)_{0}$ and $(V-I)_{0}$, and for 4 stars (for which I and $K_{\mathrm{s}}$ magnitudes are not available) $T_{\text {eff }}$ from $(B-V)_{0}$ were adopted. We checked that no significant offset exists among the different $T_{\text {eff }}$ scales: the average differences between the $T_{\text {eff }}$ scales are $T_{\mathrm{eff}}^{\left(V-K_{\mathrm{s}}\right)}-T_{\mathrm{eff}}^{(B-V)}=+49 \mathrm{~K}(\sigma=+74 \mathrm{~K}), T_{\mathrm{eff}}^{\left(V-K_{\mathrm{s}}\right)}-$ $T_{\text {eff }}^{(V-I)}=+45 \mathrm{~K}(\sigma=+51 \mathrm{~K})$, and $T_{\mathrm{eff}}^{(B-V)}-T_{\mathrm{eff}}^{(V-I)}=-30 \mathrm{~K}(\sigma=$ $+61 \mathrm{~K})$.

Gravities were derived through the Stefan-Boltzmann relation, adopting the photometric $T_{\text {eff }}$ described above, a true distance modulus of $(m-M)_{0}=13.70 \mathrm{mag}$ (Bellazzini et al. 2004), the bolometric corrections calculated according to Alonso et al. (1999), while the evolutive mass was estimated according to a grid of theoretical isochrones of different metallicities from the BaSTI database (Pietrinferni et al. 2006). Microturbulent velocities $\left(v_{\mathrm{t}}\right)$ were derived spectroscopically by erasing any trend between the line strength and iron abundance using $\sim 35-40 \mathrm{Fe} \mathrm{I}$ lines.

Chemical abundances for $\mathrm{Fe}$ and $\mathrm{Al}$ were derived with the code GALA (Mucciarelli et al. 2013) ${ }^{3}$ by comparing the theoretical and measured equivalent widths (EWs) of unblended metallic lines. The adopted model atmospheres were calculated with the last version of the code ATLAS9 ${ }^{4}$ (see Sbordone et al. 2004; Kurucz 2005). We measured the EWs with the code DAOSPEC (Stetson \& Pancino 2008) managed through the wrapper 4DAO (Mucciarelli 2013) $^{5}$. For some stars, Al lines are too weak to be

\footnotetext{
3 http://www.cosmic-lab.eu/gala/gala.php

4 http://wwwuser.oats.inaf.it/castelli/sources/ atlas9codes.html

5 http://WWW. cosmic-1ab.eu/4dao/4dao.php
} 
Table 2. Atmospheric parameters and abundance ratios (and corresponding uncertainties) for the member LRGB stars in Omega Centauri.

\begin{tabular}{lccccccc}
\hline \hline ID & $\begin{array}{c}T_{\text {eff }} \\
(\mathrm{K})\end{array}$ & $\log g$ & $\begin{array}{c}v_{\mathrm{t}} \\
\left(\mathrm{km} \mathrm{s}^{-1}\right)\end{array}$ & {$[\mathrm{Fe} / \mathrm{H}]$} & $A(\mathrm{Li})_{\mathrm{NLTE}}$ & {$[\mathrm{Na} / \mathrm{Fe}]_{\mathrm{NLTE}}$} & {$[\mathrm{Al} / \mathrm{Fe}]$} \\
\hline 22580 & 4901 & 2.31 & 1.5 & $-1.98 \pm 0.07$ & $1.02 \pm 0.05$ & $-0.42 \pm 0.08$ & $<0.94$ \\
25939 & 4890 & 2.39 & 1.6 & $-1.71 \pm 0.07$ & $1.00 \pm 0.05$ & $-0.26 \pm 0.07$ & $<0.45$ \\
31135 & 4913 & 2.36 & 1.3 & $-1.78 \pm 0.07$ & $0.85 \pm 0.05$ & $+0.18 \pm 0.06$ & $<0.57$ \\
35536 & 4907 & 2.33 & 1.5 & $-1.60 \pm 0.07$ & $0.71 \pm 0.06$ & $+0.31 \pm 0.07$ & $+1.29 \pm 0.08$ \\
45849 & 5068 & 2.41 & 1.6 & $-1.49 \pm 0.07$ & $<0.67$ & $+0.16 \pm 0.08$ & $<0.27$ \\
49844 & 4848 & 2.38 & 1.7 & $-1.67 \pm 0.07$ & $1.03 \pm 0.07$ & $-0.25 \pm 0.07$ & $<0.49$ \\
56555 & 4843 & 2.41 & 1.6 & $-1.65 \pm 0.07$ & $0.90 \pm 0.05$ & $+0.20 \pm 0.05$ & $+0.51 \pm 0.10$ \\
57780 & 4879 & 2.41 & 1.3 & $-1.87 \pm 0.07$ & $0.91 \pm 0.05$ & $-0.15 \pm 0.07$ & $<0.77$ \\
64366 & 4857 & 2.41 & 1.4 & $-1.77 \pm 0.07$ & $0.90 \pm 0.06$ & $-0.20 \pm 0.05$ & $<0.50$ \\
67162 & 4899 & 2.41 & 1.4 & $-1.54 \pm 0.07$ & $0.78 \pm 0.06$ & $+0.39 \pm 0.05$ & $+1.39 \pm 0.07$ \\
70485 & 4943 & 2.32 & 1.5 & $-1.86 \pm 0.07$ & $0.84 \pm 0.05$ & $+0.13 \pm 0.07$ & $+1.09 \pm 0.08$ \\
71476 & 4944 & 2.31 & 1.6 & $-1.80 \pm 0.07$ & $0.82 \pm 0.05$ & $+0.09 \pm 0.07$ & $+1.10 \pm 0.07$ \\
73028 & 4930 & 2.35 & 1.5 & $-1.49 \pm 0.07$ & $0.72 \pm 0.05$ & $+0.39 \pm 0.05$ & $+1.37 \pm 0.05$ \\
73743 & 4921 & 2.41 & 1.3 & $-1.84 \pm 0.07$ & $1.08 \pm 0.06$ & $+0.09 \pm 0.05$ & $<0.72$ \\
73986 & 4852 & 2.31 & 1.4 & $-1.58 \pm 0.07$ & $0.81 \pm 0.05$ & $+0.24 \pm 0.06$ & $+1.43 \pm 0.08$ \\
74878 & 4927 & 2.36 & 1.5 & $-1.81 \pm 0.07$ & $1.02 \pm 0.06$ & $+0.07 \pm 0.07$ & $<0.59$ \\
77093 & 4918 & 2.38 & 1.4 & $-1.85 \pm 0.09$ & $1.04 \pm 0.07$ & $-0.29 \pm 0.09$ & $<0.77$ \\
\hline
\end{tabular}

Notes. This table is available in its entirety at the CDS.

measured and we computed upper limits adopting as EW three times the uncertainty calculated according to the Cayrel (1988) formula.

We derived abundances for $\mathrm{Li}$ and $\mathrm{Na}$ through a $\chi^{2}$ minimization, performed with our own code SALVADOR, between the observed and synthetic spectra. The synthetic spectra were computed with the code SYNTHE (Sbordone et al. 2004; Kurucz 2005), including all the atomic and molecular transitions from the Kurucz/Castelli linelist. We derived the Li abundances from the $\mathrm{Li}$ resonance doublet at $\sim 6708 \AA$, including the corrections for non-local thermodynamical equilibrium from Lind et al. (2008). Lithium abundances were derived for 168 targets, while for 33 stars the Li line is too weak and only upper limits are provided. $\mathrm{Na}$ abundances from the $\mathrm{Na} \mathrm{D}$ doublet at 5890-5896 $\AA$ were corrected for departures from local thermodynamical equilibrium adopting the corrections computed by Lind et al. (2011).

We calculated abundance uncertainties by adding in quadrature the errors arising from the measurement procedure (EW or spectral fitting) and those arising from the adopted atmospheric parameters; uncertainties in $T_{\text {eff }}$ and $\log g$ were added directly because the two parameters are correlated. For Fe and $\mathrm{Al}$, we consider as internal error the dispersion normalized to the root mean square of the number of used lines. For $\mathrm{Li}$ and $\mathrm{Na}$ abundances, derived from spectral synthesis, we estimated the uncertainties in the fitting procedure resorting to Monte Carlo simulations. For each star, a sample of 300 noisy spectra was created, injecting Poissonian noise in the best-fit synthetic spectrum (rebinning to the FLAMES-GIRAFFE pixel size). Each sample of spectra was analysed with the same procedure adopted for the real spectra. The dispersion of the derived abundance distribution was assumed as $1 \sigma$ uncertainty in the abundance. Uncertainties due to the atmospheric parameters were derived by repeating the analysis varying each time one only parameter by the corresponding uncertainty $\left(\sigma_{T_{\mathrm{eff}}}= \pm 50 \mathrm{~K}, \sigma_{\mathrm{v}_{\mathrm{t}}}=\right.$ $\pm 0.1 \mathrm{~km} \mathrm{~s}^{-1}, \sigma_{\log g}= \pm 0.1$ ).

Typical formal uncertainties in $A(\mathrm{Li})$ are of the order of $0.05-$ 0.07 dex because the uncertainty in $T_{\text {eff }}$ is the dominant source of error for these abundances, while the error due to the fitting procedure is smaller than 0.03 dex because of the high $\mathrm{S} / \mathrm{N}$ of the spectra. Atmospheric parameters, abundance ratios, and their uncertainties for all the member stars are listed in Table 2.

\section{4. $[\mathrm{Fe} / \mathrm{H}]$ distribution}

Figure 2 shows the $[\mathrm{Fe} / \mathrm{H}]$ distribution of the entire sample (upper panel) as a generalized histogram, i.e. a representation that removes the effects due to the choice of the starting point and bin size by taking the uncertainties in each individual value into account (see Laird et al. 1988).

The iron distribution ranges from $-2.06 \mathrm{dex}$ to $-0.76 \mathrm{dex}$ with a main peak at $[\mathrm{Fe} / \mathrm{H}] \sim-1.85 \mathrm{dex}$, a clear second peak at $[\mathrm{Fe} / \mathrm{H}] \sim-1.55 \mathrm{dex}$, a long metal-rich tail with a clump of stars at $[\mathrm{Fe} / \mathrm{H}] \sim-0.9 \mathrm{dex}$, and a possible small peak at $[\mathrm{Fe} / \mathrm{H}] \sim-1.3 \mathrm{dex}$. This distribution is qualitatively similar to those previously discussed in other studies (Johnson et al. 2008, 2009; Johnson \& Pilachowski 2010; Marino et al. 2011). The lower panel of Fig. 2 shows the $[\mathrm{Fe} / \mathrm{H}]$ distribution by Johnson \& Pilachowski (2010) that presented the largest (855 stars) dataset of abundances for RGB stars of Omega Centauri. We note that the main peak of our distribution is slightly more metal poor than that by Johnson \& Pilachowski (2010). We estimate a difference of -0.08 dex between the median values of the metal-poor components of the two distributions. In Fig. 2 the $[\mathrm{Fe} / \mathrm{H}]$ distribution by Johnson \& Pilachowski (2010) has been shifted by -0.08 dex to match the main peaks of the two distributions. Because there are no stars in common with this study (that is focussed on RGB stars brighter than the RGB bump), we cannot directly investigate the origin of this small difference. However, we noted that the $V$-band magnitudes used by Johnson \& Pilachowski (2010) are brighter than those of Bellini et al. (2009) by about $0.1 \mathrm{mag}$ (while the $K_{\mathrm{s}}$-band magnitudes are the same), leading to slightly hotter $T_{\text {eff }}$ with respect to our values. Despite this small offset, the two distributions are very similar. Following the scheme used by Johnson \& Pilachowski (2010) to describe their $[\mathrm{Fe} / \mathrm{H}]$ distribution, we adopted the nomenclature proposed by Sollima et al. (2005a) to associate the individual RGBs observed in the CMD 


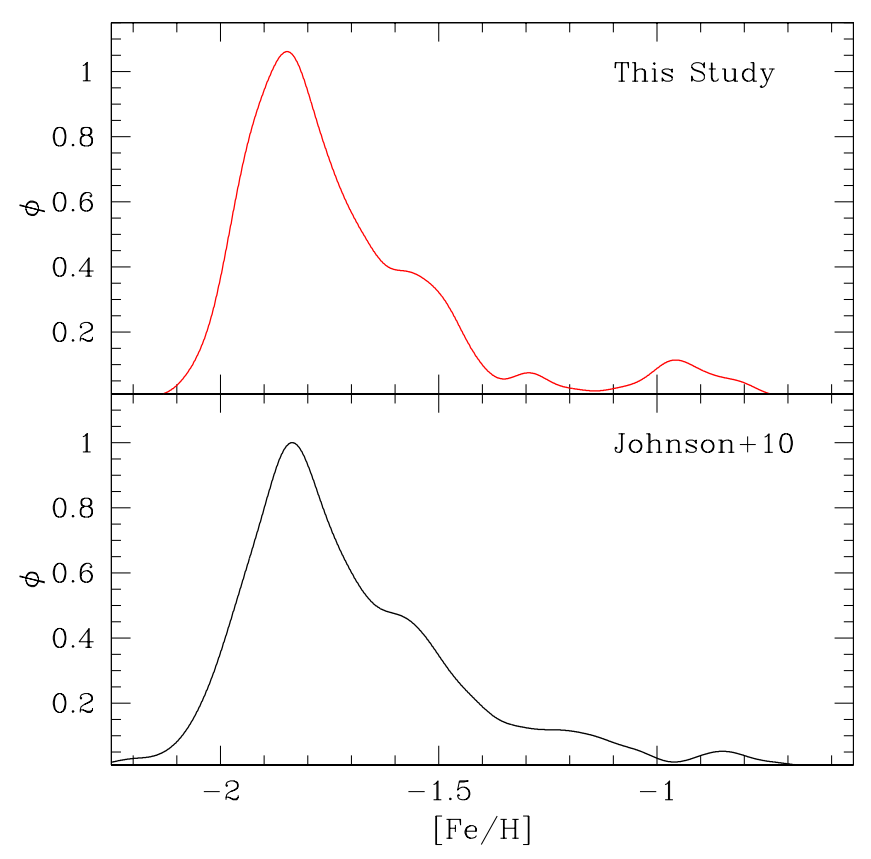

Fig. 2. Generalized histograms for the LRGB stars of Omega Centauri discussed in this study (upper panel) and for the bright RGB stars discussed by Johnson \& Pilachowski (2010), shifted by -0.08 dex to match the main peaks of the two distributions.

of Omega Centauri with the components of its iron distribution. The main peak in our $[\mathrm{Fe} / \mathrm{H}]$ distribution can be associated with the RGB-MP (the main giant branch observed in the CMD of Omega Centauri), the second peak to the RGBInt1, while the most metal-rich peak to the anomalous RGB. Sollima et al. (2005a) identified two other RGBs with metallicites intermediate between those of RGB-Int1 and the anomalous RGB. Johnson \& Pilachowski (2010) identified the stars in the range $[\mathrm{Fe} / \mathrm{H}] \sim-1.4 /-1.0$ dex in their $[\mathrm{Fe} / \mathrm{H}]$ distribution as belonging to the RGB-Int2 and RGB-Int3. In our distribution this $[\mathrm{Fe} / \mathrm{H}]$ range is less populated with respect to the Johnson \& Pilachowski (2010) distribution, probably because of a poor sampling of the reddest stars; our targets are confined to a narrow strip on the CMD (see Fig. 1).

\section{Lithium in Omega Centauri}

We discuss the $A(\mathrm{Li})$ distribution in 199 LRGB stars of Omega Centauri, excluding two targets that reveal a high Li content and that will be discussed in a forthcoming paper.

The upper panel of Fig. 3 shows the behaviour of $A(\mathrm{Li})$ as a function of $[\mathrm{Fe} / \mathrm{H}]$ for the true measures (166 stars, gray circles) and the upper limits (33 stars, red arrows). Considering only the true measures, the $A(\mathrm{Li})$ distribution ranges from 0.47 to $1.19 \mathrm{dex}$. The $A(\mathrm{Li})$ distribution clearly peaks at $A(\mathrm{Li}) \sim 1$ dex but with the presence of a significant percentage of stars with $A(\mathrm{Li})<0.8$ dex. Stars with $A(\mathrm{Li}) \sim 1$ dex are found only at $[\mathrm{Fe} / \mathrm{H}]<-1.3 \mathrm{dex}$, while Li-poor stars are found at all the metallicities. In particular, the $A(\mathrm{Li})$ distribution for stars with $[\mathrm{Fe} / \mathrm{H}]<-1.6 \operatorname{dex}$ (corresponding to the main population of Omega Centauri, RGB-MP) is dominated by stars with $A(\mathrm{Li}) \sim 1 \mathrm{dex}$. In the $[\mathrm{Fe} / \mathrm{H}]$ range between -1.6 and $-1.3 \mathrm{dex}$ (likely connected to RGB-Int1) the distribution is dominated by stars with $A(\mathrm{Li})<0.8$ dex, while the component at $A(\mathrm{Li}) \sim 1$ dex decreases in number. For $[\mathrm{Fe} / \mathrm{H}]>-1.3$ dex the component with
$A(\mathrm{Li}) \sim 1$ dex totally disappears and all stars have $A(\mathrm{Li})$ lower than $\sim 0.6-0.7 \mathrm{dex}$.

The lower panel of Fig. 3 shows the run of the ratio of the number of Li-rich to Li-poor stars (defined assuming $A(\mathrm{Li})=0.8 \mathrm{dex}$ as threshold) as a function of the iron abundance. The data were grouped into four metallicity bins, chosen according to the metallicity distribution (Fig. 2) centred at $[\mathrm{Fe} / \mathrm{H}]=$ $-1.85,-1.55,-1.30$, and $-0.9 \mathrm{dex}$, and with width of $0.45,0.20$, 0.34 , and $0.3 \mathrm{dex}$. The stars with $[\mathrm{Fe} / \mathrm{H}]<-1.65$ dex show a constant number ratio that drops at higher metallicity, reaching 0 for the most metal-rich stars where all the stars have $A(\mathrm{Li})<0.8$.

In order to assess whether the lack of Li-rich stars in the metal-rich regime is real or due to small number statistics, we performed a Monte Carlo simulation from a $A(\mathrm{Li})$ distribution resembling that observed for stars with $[\mathrm{Fe} / \mathrm{H}]<-1.6 \mathrm{dex}$. We extracted from this distribution 10000 samples of 40 stars and 10000 samples of 15 stars, corresponding to the number of stars observed in the third and forth metallicity bin, respectively. We found that the probability to observe a $N_{\mathrm{Li} \text {-rich }} / N_{\mathrm{Li} \text {-poor }}$ number ratio smaller than 0.5 is 0 and less than $0.03 \%$ for the two samples, ruling out that the observed lack of stars with $A(\mathrm{Li}) \sim 1 \mathrm{dex}$ is due to the small number of stars. Also, we checked that different choices for the metallicity bins do not change this conclusion. Hence, the difference between the $A(\mathrm{Li})$ distributions of metal-poor and metal-rich stars is real.

The peak of the number distribution of $A(\mathrm{Li})$ for the metalpoor stars well agrees with the $A(\mathrm{Li})$ abundances previously measured in Population II LRGB stars, i.e. in Milky Way field stars (Mucciarelli et al. 2012), Galactic GCs (Lind et al. 2009; Mucciarelli et al. 2011; D'Orazi et al. 2014, 2015) and the extragalactic GC M54 (Mucciarelli et al. 2014). This finding confirms the previous result (based on dwarf stars) by Monaco et al. (2010) that Omega Centauri formed with the same lithium content of other Population II stars, formed in the Milky Way. Considering only the stars with $[\mathrm{Fe} / \mathrm{H}]<-1.3 \mathrm{dex}$, the main peak of the $A(\mathrm{Li})$ number distribution $(A(\mathrm{Li})>0.8 \mathrm{dex})$ includes about $60 \%$ of the stars. This fraction well matches the fraction of the true measures of $A(\mathrm{Li})$ among dwarf stars of Omega Centauri analysed by Monaco et al. (2010) in a similar range of metallicity $([\mathrm{Fe} / \mathrm{H}]<-1.4 \mathrm{dex})$.

The most interesting feature in this $A(\mathrm{Li})$ distribution is the lack of stars with $A(\mathrm{Li}) \sim 1$ dex at metallicities higher than $[\mathrm{Fe} / \mathrm{H}] \sim-1.3$ dex. The blue line shown in Fig. 3 is the expected run of $A(\mathrm{Li})$ with $[\mathrm{Fe} / \mathrm{H}]$ in $\mathrm{LRGB}$ stars assuming that they are all born with the same initial $A(\mathrm{Li})$, according to models from Mucciarelli et al. (2012) and assuming an age of 12.5 Gyr. In particular, we assumed an initial value able to match the observed $A(\mathrm{Li})$ of the peak of the number distribution of the metal-poor component; however the shape of the curve is independent of the assumed value for the initial $A(\mathrm{Li})$. The model predicts that $A(\mathrm{Li})$ in LRGB stars mildly decreases over a large range of $[\mathrm{Fe} / \mathrm{H}]$ and should only rapidly drop because of the deeper convective envelope of such metal-rich stars for $[\mathrm{Fe} / \mathrm{H}]$ $>-0.9$ dex. If we assume that all the stars in Omega Centauri formed with the same initial $\mathrm{Li}$ content, a clear drop of the $\mathrm{Li}$ abundance is expected at $[\mathrm{Fe} / \mathrm{H}]>-0.9 /-0.8$ dex. Instead, the stars of Omega Centauri with $[\mathrm{Fe} / \mathrm{H}]>-1.3 \mathrm{dex}$ are systematically depleted in lithium. We recall that LRGB stars of GCs with $[\mathrm{Fe} / \mathrm{H}] \sim-1.1 /-1.2$ dex (see e.g. M4, Mucciarelli et al. 2011; Monaco et al. 2012 and NGC 2808, D'Orazi et al. 2015) have $A(\mathrm{Li})$ compatible with that measured in metal-poor clusters.

We investigated whether other parameters can affect the behaviour of $A(\mathrm{Li})$ in these stars, in particular the helium mass 


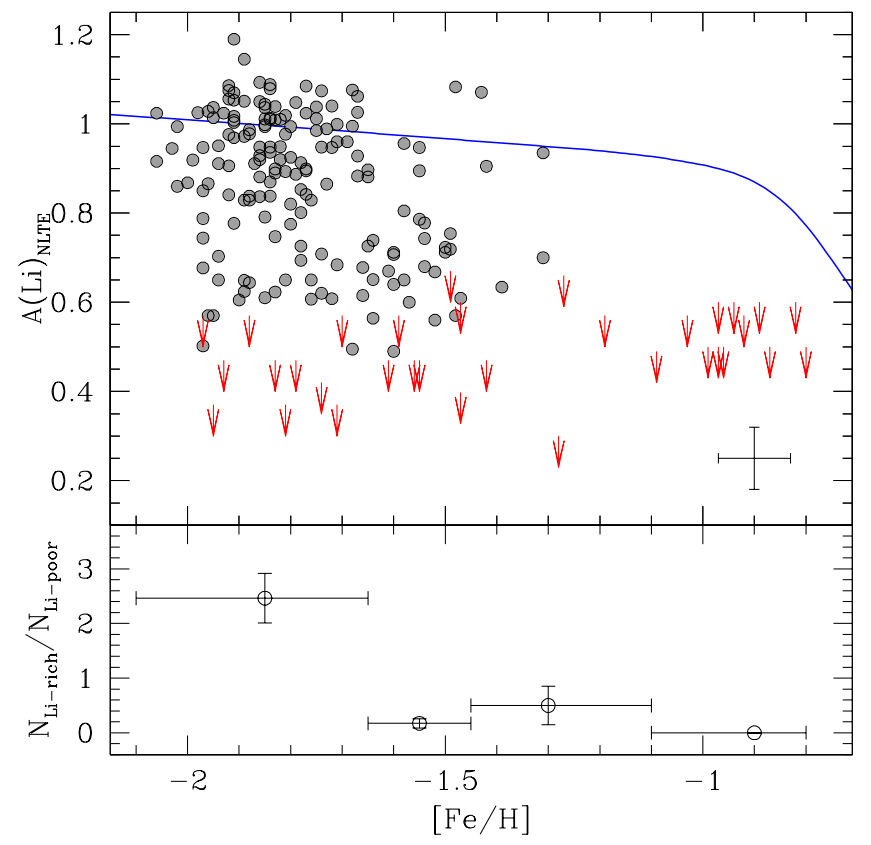

Fig. 3. Upper panel: behaviour of $A(\mathrm{Li})$ as a function of $[\mathrm{Fe} / \mathrm{H}]$. The gray points indicate the true measures; red arrows indicate the upper limits. The blue line shows the theoretical behaviour of $A(\mathrm{Li})$ as a function of $[\mathrm{Fe} / \mathrm{H}]$ in LRGB stars starting from the same initial $A(\mathrm{Li})$. Lower panel: behaviour of the fraction of number of stars with $A(\mathrm{Li})>0.8$ and with $A(\mathrm{Li})<0.8$ as a function of $[\mathrm{Fe} / \mathrm{H}]$ computed for four metallicity bins. Vertical error bars represent the uncertainties in the number ratio, the horizontal error bars denote the corresponding metallicity range.

fraction $\mathrm{Y}$ and stellar age. Several photometric observations (such as the extended blue horizontal branch and splitting of the main sequence) reveal a large spread in the initial He content of Omega Centauri stars. In particular, the most metalrich populations were suggested to have a helium mass fraction up to $Y \sim 0.35$ (Norris 2004; Piotto et al. 2005; Sollima et al. 2005b; Renzini 2008). We checked that for metallicity $[\mathrm{M} / \mathrm{H}]=$ -1.01 dex, an increase of $Y$ from 0.24 up to 0.35 changes the value of $A(\mathrm{Li})$ in LRGB stars by only 0.03 dex.

The precise age spread in Omega Centauri is still debated and the metal-rich populations are proposed to be coeval to the main population or significantly younger by some Gyr (see e.g. Ferraro et al. 2004; Freyhammer et al. 2005; Villanova et al. 2014; Tailo et al. 2016). However, a decrease of the age from $12.5 \mathrm{Gyr}$ to $8.5 \mathrm{Gyr}$ (both adopting $Y=0.25$ and $Y=0.35$ ) leads to a decrease of $A(\mathrm{Li})$ by $\sim 0.05-0.06 \mathrm{dex}$ with respect to the value calculated for $12.5 \mathrm{Gyr}$ and $Y=0.25$. Therefore, an increase of the He content and/or a decrease of the age cannot explain the drop of $A(\mathrm{Li})$ observed for $[\mathrm{Fe} / \mathrm{H}]>$ -1.3 dex.

Finally, we note that the model predictions for the surface $A(\mathrm{Li})$ of the observed RGB stars (blue line in Fig. 3) include potential surface Li depletion during the MS due to burning at the bottom of the convective envelopes.

\section{Li-Na anti-correlation}

The comparison between $\mathrm{Li}$ and $\mathrm{Na}$ abundances provides important clues to understand the nature of the Li-poor stars observed at all the metallicities in Omega Centauri. This stellar system has a wide range of $[\mathrm{Na} / \mathrm{Fe}]$ from $[\mathrm{Na} / \mathrm{Fe}]$ $\sim-0.5$ dex to $\sim+0.6$ dex. We note that our $[\mathrm{Na} / \mathrm{Fe}]$ distribution

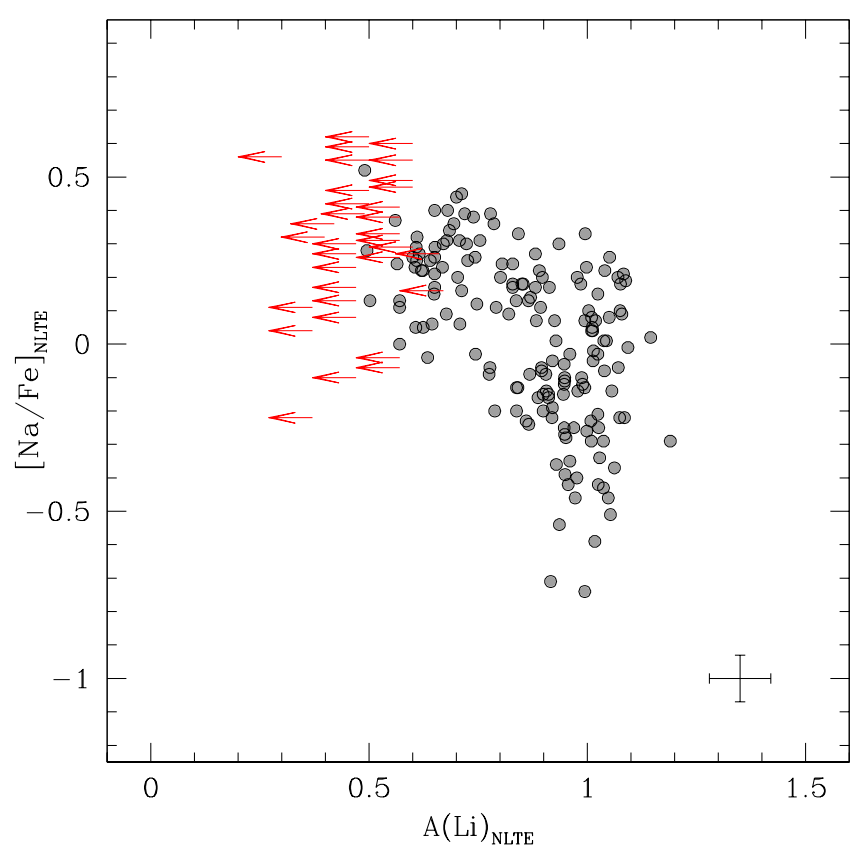

Fig. 4. Behaviour of $[\mathrm{Na} / \mathrm{Fe}]$ as a function of $A(\mathrm{Li})$ for the entire spectroscopic sample studied in this work (gray circles). Red arrows indicate the upper limits for $A(\mathrm{Li})$.

is shifted towards lower values with respect to those measured by Johnson \& Pilachowski (2010) and Marino et al. (2011) because these two works do not take into account departures from the local thermodynamical equilibrium (the corrections for the $\mathrm{Na}$ abundances are usually negative). Figures 4 and 5 show the run of $[\mathrm{Na} / \mathrm{Fe}]$ and $[\mathrm{Al} / \mathrm{Fe}]$ as a function of $A(\mathrm{Li})$, respectively. The red arrows represent the upper limits for $\mathrm{Li}$ and the blue arrows indicate the upper limits for $[\mathrm{Al} / \mathrm{Fe}]$. In both plots a clear anticorrelation is found; the stars with the higher $A(\mathrm{Li})$ cover a large range of $[\mathrm{Na} / \mathrm{Fe}]$ and $[\mathrm{Al} / \mathrm{Fe}]$, while the stars with $A(\mathrm{Li})$ lower than $\sim 0.8$ dex have systematically higher $[\mathrm{Na} / \mathrm{Fe}]$ and $[\mathrm{Al} / \mathrm{Fe}]$ abundances. Direct evidence of this behaviour is shown in Fig. 6, where the spectra of two stars stars with similar atmospheric parameters and metallicity are compared (namely \#206770 and \#213229, red and black curves, respectively). As clearly visible, the spectrum of \#213229 shows $\mathrm{Al}$ and $\mathrm{Na}$ lines stronger than those of \#206770 (where the Al lines are totally lacking), but also an undetectable $\mathrm{Li}$ line, clearly visible in the other spectrum.

The Li-Na anti-correlation is visible only in the metal-poor stars because the presence of a significant star-to-star $A(\mathrm{Li})$ scatter is restricted to $[\mathrm{Fe} / \mathrm{H}]<-1.3 \mathrm{dex}$ (see Fig. 3). In particular, the stars with low $A(\mathrm{Li})$ are all significantly enriched in $\mathrm{Na}$, even if other stars with similar high $\mathrm{Na}$ have normal $A(\mathrm{Li})$. Figure 7 shows the behaviour of $[\mathrm{Na} / \mathrm{Fe}]$ as a function of $[\mathrm{Fe} / \mathrm{H}]$ for our sample (a similar run has also been found by Johnson \& Pilachowski 2010; Marino et al. 2011). [ $\mathrm{Na} / \mathrm{Fe}]$ increases with the iron content and the higher $\mathrm{Na}$ abundances are measured in the most metal-rich stars. Hence, stars with $[\mathrm{Fe} / \mathrm{H}]$ $>-1.3$ dex are all depleted in Li but enriched in $\mathrm{Na}$.

We compare the Li-Na distribution of Omega Centauri with those measured in mono-metallic GCs, namely NGC 6397, NGC 6752, M4, and 47 Tucanae. The distributions of $\mathrm{Li}-\mathrm{Na}$ of these clusters are shown in Fig. 8 in comparison with that of the LRGB stars in Omega Centauri: light gray points and arrows show the entire sample of target stars studied, while dark 


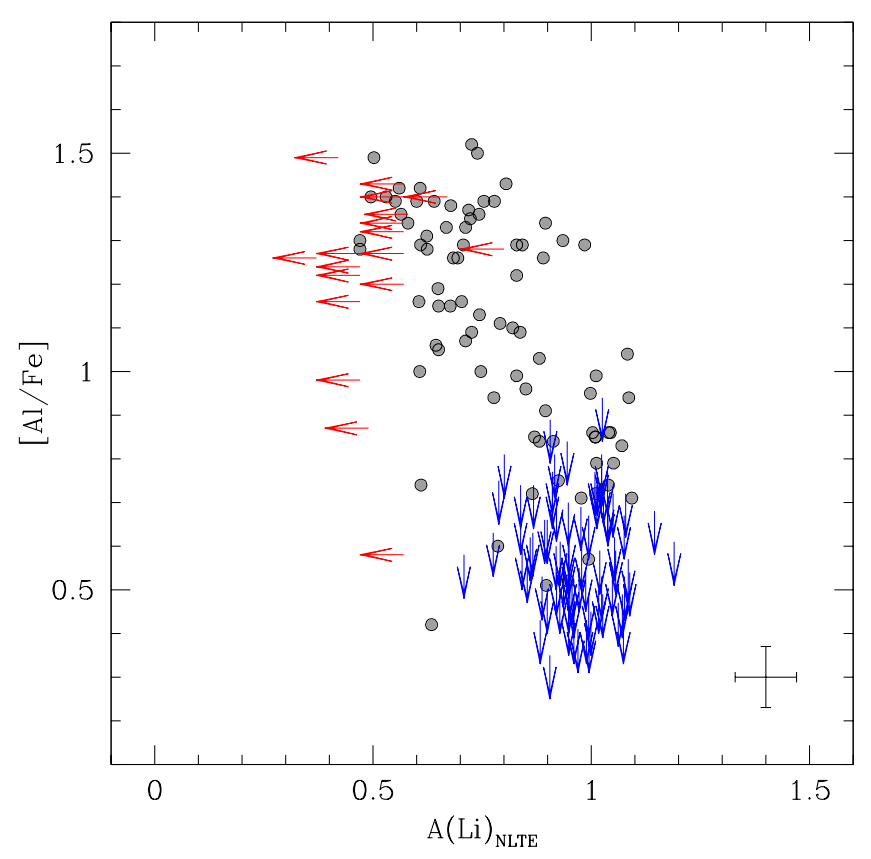

Fig. 5. Behaviour of $[\mathrm{Al} / \mathrm{Fe}]$ as a function of $A(\mathrm{Li})$ for the entire spectroscopic sample studied in this work (gray circles). Red arrows indicate the upper limits for $A(\mathrm{Li})$, blue arrows show the upper limits for $\mathrm{Al}$.
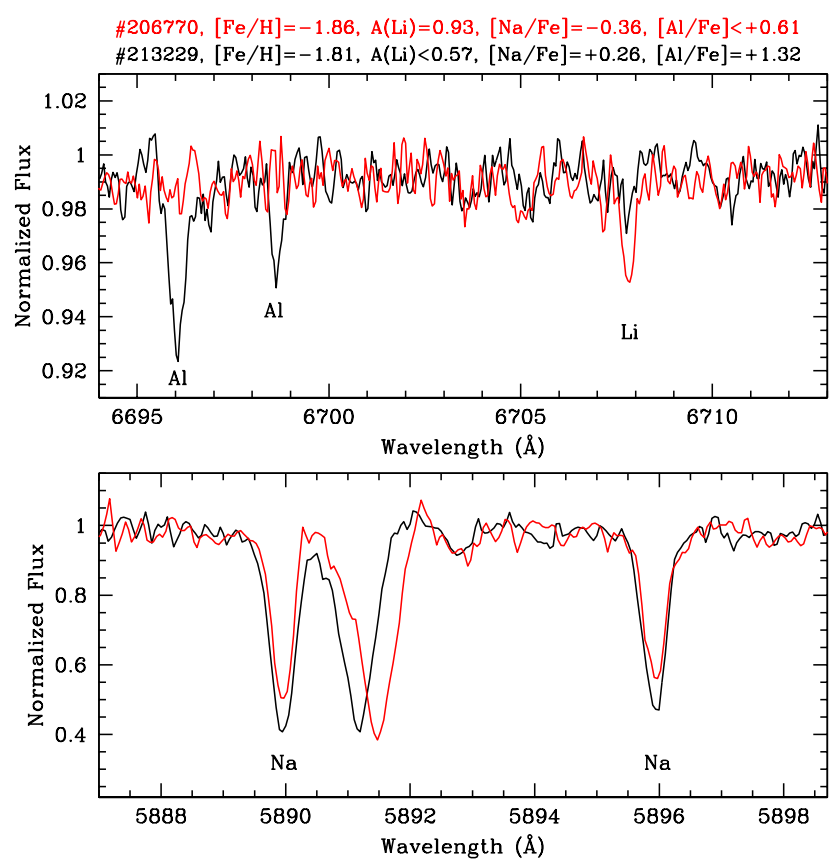

Fig. 6. Comparison between two stars of Omega Centauri, namely \#206770 and \#213229 (red and black curves respectively). Showing these two stars have similar atmospheric parameters and metallicity but different depths for $\mathrm{Li}, \mathrm{Na}$, and $\mathrm{Al}$ lines. The two strong lines at $~ 5891 \AA$ visible in the lower panel and shifted each other are Na interstellar lines.

gray points and arrows show the target stars of Omega Centauri selected with metallicities close to those of the comparison GCs included in each panel. Because the Li abundances in these GCs are measured in dwarf stars we shifted these abundances in order to match the median $A(\mathrm{Li})$ of each cluster with the peak of the $A(\mathrm{Li})$ distribution of Omega Centauri, which is lower owing to the effect of the FDU.

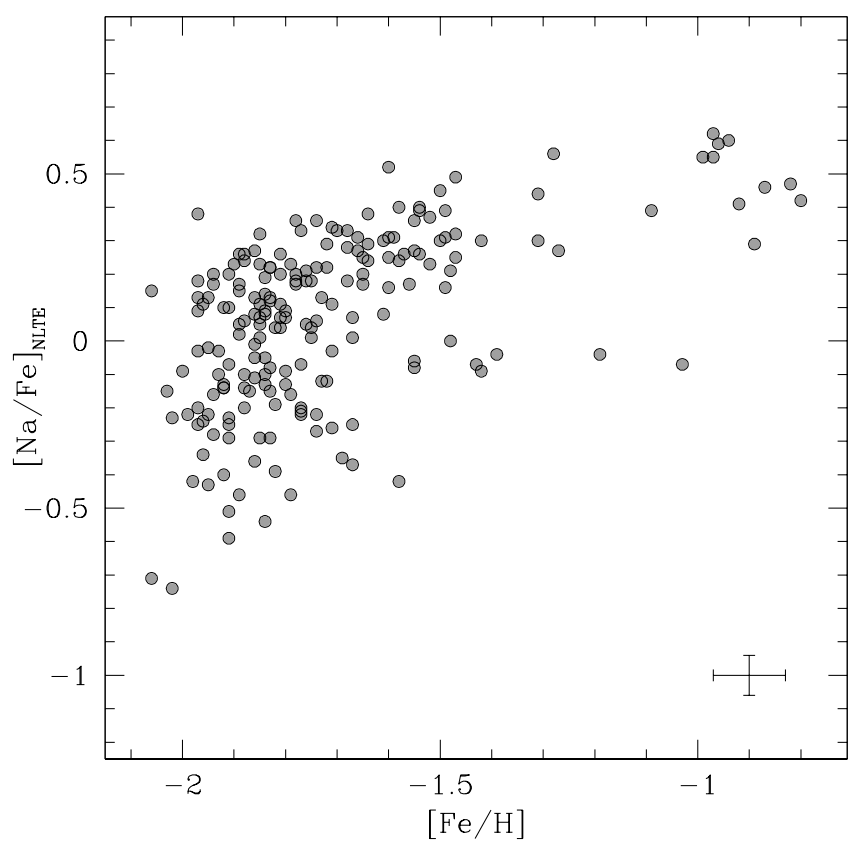

Fig. 7. Behaviour of $[\mathrm{Na} / \mathrm{Fe}]$ as a function of $[\mathrm{Fe} / \mathrm{H}]$.

In NGC 6397 (blue circles in Fig. 8) most of the 100 dwarf stars studied by Lind et al. (2009) have a similar $A(\mathrm{Li})$ but over a large range of $\mathrm{Na}$ (hence corresponding to $1 \mathrm{P}$ and $2 \mathrm{P}$ stars). Among these 100 targets, three stars have $A(\mathrm{Li})$ lower than the other stars by $\sim 0.4-0.5$ dex and they have the highest values of $[\mathrm{Na} / \mathrm{Fe}]$ of the sample.

NGC 6752 (green circles) shows a clear Li-Na anticorrelation (Pasquini et al. 2005) where the most Li-poor stars have $A(\mathrm{Li})$ lower by $\sim 0.4$ dex with respect to the most Li-rich stars. At variance with NGC 6397 (and also Omega Centauri) where most of the stars exhibit a large $\mathrm{Na}$ spread but similar $A(\mathrm{Li})$, in NGC 6752 the $\mathrm{Na}$ and $\mathrm{Li}$ abundances in the studied stars follow a linear behaviour. Even if the sample discussed by Pasquini et al. (2005) includes only 9 dwarf stars, its large spread in $A(\mathrm{Li})$ has been confirmed by Shen et al. (2010) with a sample of 112 dwarf stars (and where a $\mathrm{Li}-\mathrm{O}$ correlation has been detected).

Monaco et al. (2012) present $\mathrm{Na}$ and $\mathrm{Li}$ abundances in 70 dwarf stars in M4 (red triangles in Fig. 8), excluding one Lirich star. The $\mathrm{Na}-\mathrm{Li}$ distribution in M4 is similar to that of NGC 6397, with most of the stars with similar Li content and different $[\mathrm{Na} / \mathrm{Fe}]$ but a larger star-to-star scatter in $A(\mathrm{Li})$ among the $2 \mathrm{P}$ stars. We note that the most Li-poor star in this sample has a $A(\mathrm{Li})$ lower by $\sim 0.3$ dex than the median $A(\mathrm{Li})$ value and it is among the most Na-rich stars.

47Tucanae (cyan squares in Fig. 8) exhibits a large starto-star scatter in its $\mathrm{Li}$ content. D'Orazi et al. (2010) and Dobrovolskas et al. (2014) from the analysis of 110 dwarf stars in this cluster do not find clear evidence of $\mathrm{Li}-\mathrm{Na}$ anticorrelation, while a hint of $\mathrm{Li}-\mathrm{O}$ correlation is detected.

Other GCs have been investigated by D'Orazi et al. (2014, 2015), who discuss the behaviour of $A(\mathrm{Li})$ in LRGB stars as a function of $\mathrm{Al}$ abundance. In most of these GCs (namely NGC 362, NGC 1904, NGC 5904, and NGC 6218) all the stars have the same $A(\mathrm{Li})$ regardless of the $\mathrm{Al}$ abundance. On the other hand, in NGC 2808 some Al-rich LRGB stars have A(Li) lower by $\sim 0.3 \mathrm{dex}$ than that in other stars with similar $\mathrm{Al}$ content. 


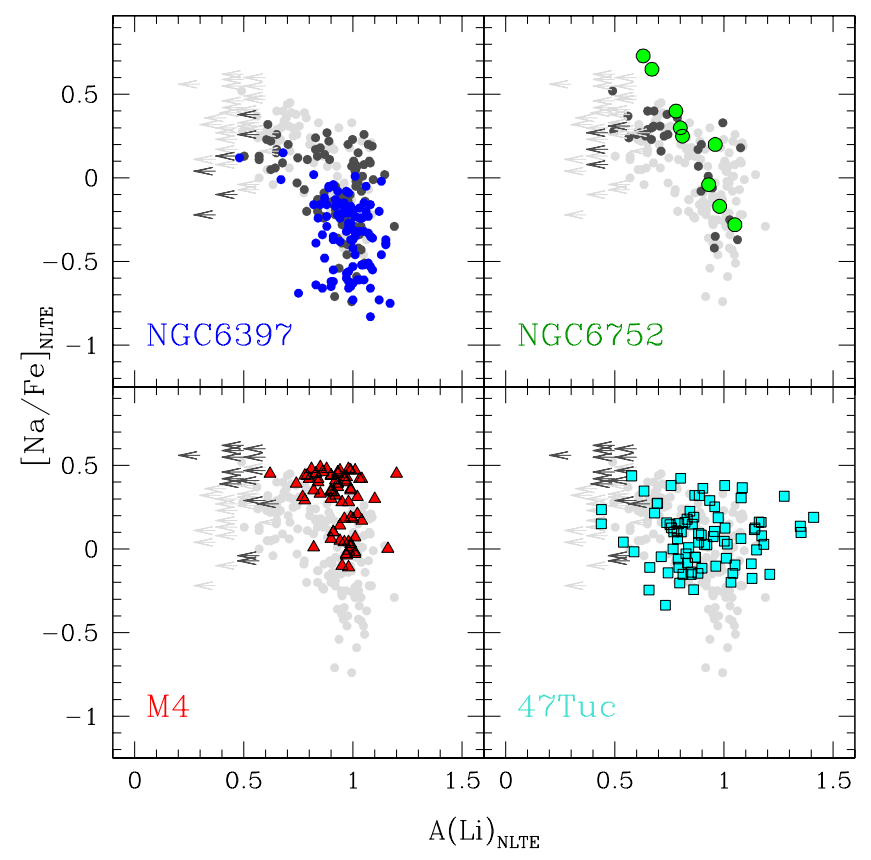

Fig. 8. Comparison between the $[\mathrm{Na} / \mathrm{Fe}]-A(\mathrm{Li})$ distribution of the stars in Omega Centauri (gray points and arrows) and the abundances measured in dwarf stars of some mono-metallic GCs from the literature, namely NGC 6397 (blue circles; Lind et al. 2009), NGC 6752 (green circles; Pasquini et al. 2005), M4 (red triangles; Monaco et al. 2012) and 47 Tucanae (cyan squares; Dobrovolskas et al. 2014). Light gray points and arrows indicate the entire sample of stars in Omega Centauri studied; dark gray points and arrows represent the target stars selected with a metallicity similar to that of the reference Galactic GC shown in each panel.

\section{Summary and conclusions}

We derived surface lithium abundances for 199 LRGB stars members of the stellar system Omega Centauri and distributed over its entire metallicity range. The main results are as follows:

- The $A(\mathrm{Li})$ number distribution of LRGB stars in Omega Centauri is peaked at $A(\mathrm{Li}) \sim 1 \mathrm{dex}$. This value is compatible with the abundances measured in LRGB stars belonging to other GCs and in metal poor field stars and this abundance can be considered as the normal $A(\mathrm{Li})$ in Population II LRGB stars. Additionally, the $A(\mathrm{Li})$ distribution of Omega Centauri shows also a prominent $\mathrm{Li}$-poor tail $(A(\mathrm{Li}) \lesssim 0.8 \mathrm{dex})$.

- The stars with normal $A(\mathrm{Li})$ are found at $[\mathrm{Fe} / \mathrm{H}]<-1.3 \mathrm{dex}$, while the Li-poor stars are found at all the metallicities. All the stars with $[\mathrm{Fe} / \mathrm{H}]>-1.3$ dex exhibit low $A(\mathrm{Li})$ values.

- A clear $\mathrm{Li}-\mathrm{Na}$ anti-correlation is found. The stars with normal $A(\mathrm{Li})$ cover a large range of $[\mathrm{Na} / \mathrm{Fe}] ; A(\mathrm{Li}) \lesssim 0.8 \mathrm{dex}$ are characterized by enhanced values of $[\mathrm{Na} / \mathrm{Fe}]$. Similarly, a $\mathrm{Li}-\mathrm{Al}$ anti-correlation is detected.

The $A(\mathrm{Li})$ distribution in Omega Centauri is more complex than those observed in mono-metallic GCs, reflecting the peculiar (and not yet totally understood) chemical evolution of this system. We can draw a qualitative scheme to describe this distribution, classifying the stars according to their, $\mathrm{Li}, \mathrm{Na}$, and $\mathrm{Fe}$ abundances. In particular, we used $[\mathrm{Na} / \mathrm{Fe}]$ to discriminate between $1 \mathrm{P}($ low $[\mathrm{Na} / \mathrm{Fe}])$ and $2 \mathrm{P}($ high $[\mathrm{Na} / \mathrm{Fe}])$ stars. We identify four main groups of stars, sketched in Fig. 9:

1. 1P stars $([\mathrm{Fe} / \mathrm{H}]<-1.3$ dex $)$, with low $[\mathrm{Na} / \mathrm{Fe}]$ and normal Li.

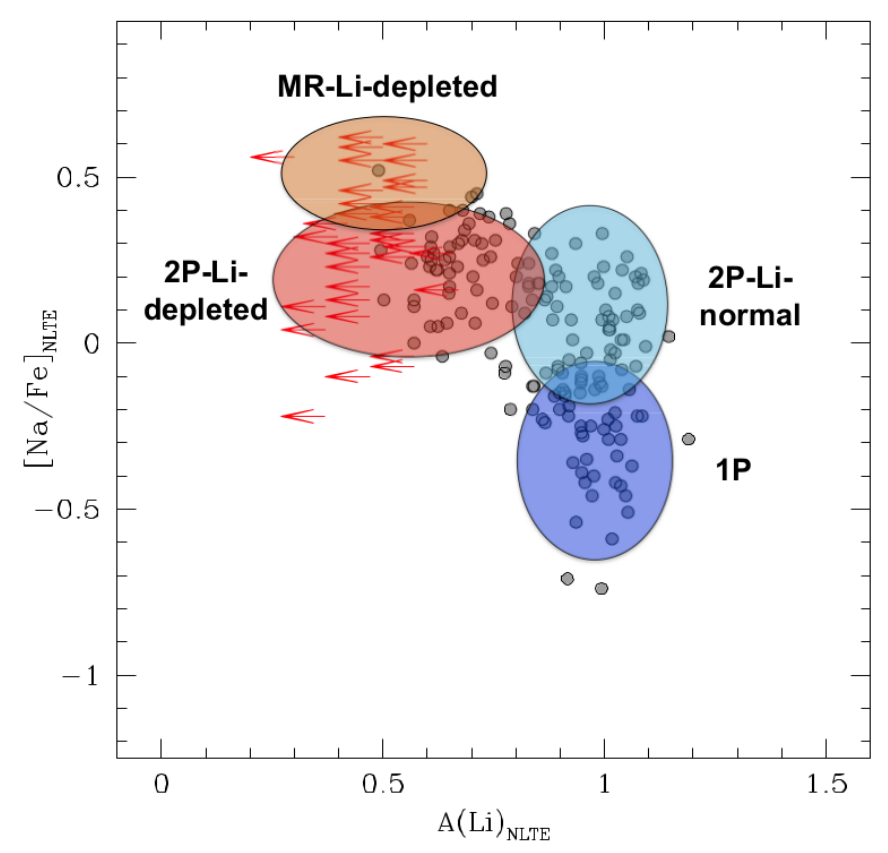

Fig. 9. Same as Fig. 4 but with superimposed the mean loci for the proposed classification of the Omega Centauri's stars according to their $\mathrm{Li}$ and $\mathrm{Na}$ abundances.

2. $2 \mathrm{P}$ stars $([\mathrm{Fe} / \mathrm{H}]<-1.3 \mathrm{dex})$ enriched in $[\mathrm{Na} / \mathrm{Fe}]$ and with the same (normal) $A(\mathrm{Li})$ measured in $1 \mathrm{P}$ stars; hereafter we refer to these stars as $2 P$-Li-normal.

3. $2 \mathrm{P}$ stars $([\mathrm{Fe} / \mathrm{H}]<-1.3$ dex $)$ enriched in $[\mathrm{Na} / \mathrm{Fe}]$ similar to the $2 P$-Li-normal stars, but depleted $\mathrm{Li}(A(\mathrm{Li}) \lesssim 0.8 \mathrm{dex})$ and referred to in this work as $2 P$-Li-depleted.

4. Stars with $[\mathrm{Fe} / \mathrm{H}]>-1.3$ dex (labelled as MR-Li-depleted) that have $A(\mathrm{Li}) \lesssim 0.6 \mathrm{dex}$ and high $[\mathrm{Na} / \mathrm{Fe}]$ abundances. In particular, the $[\mathrm{Na} / \mathrm{Fe}]$ abundance of these stars is slightly higher than that of $2 \mathrm{P}$ stars because the $[\mathrm{Na} / \mathrm{Fe}]$ increases with the metallicity (see Fig. 7 and also Johnson \& Pilachowski 2010; Marino et al. 2011).

The classification proposed in Fig. 9 only provides a simple guideline to highlight the complexity of the $\mathrm{Li}-\mathrm{Na}$ distribution in this system, and the main features to be explained by models for the formation of Omega Centauri. It does not imply a specific formation timeline.

The overall picture is that mono-metallic GCs (see Fig. 8) show, to a lesser extent, the same sub-populations identified in the metal-poor stars of Omega Centauri. These clusters are dominated by $1 \mathrm{P}$ and $2 P$-Li-normal stars, with the presence of a minor component of Na-rich, Li-poor stars (even if the identification of a clear boundary between $2 P$-Li-normal and $2 P$-Li-depleted populations is not trivial). On the other hand, the fraction of $2 P$ Li-depleted stars observed in Omega Centauri $(\sim 30 \%)$ turns out to be exceptional with respect to the other GCs, where the fraction of these stars is about 3-5\%.

Within the canonical views of the formation of multiple populations, 1P stars would correspond to the first stellar generation and we can consider their surface $A(\mathrm{Li})$ as a good tracer of the initial lithium content of the stellar system (after the effect of the FDU is taken into account). The high $[\mathrm{Na} / \mathrm{Fe}]$ measured in the other (2P) stars indicates that these stars formed from a gas enriched by the products of proton-capture cycles (occurring at temperatures higher than that of Li burning). The timeline of the formation of the 2P stars (both with normal and depleted Li content) in Omega Centauri depends on the chemical evolution of 
the system and the role played by the different polluter stars. The 2P-Li-depleted stars may have formed from a Li-poor or $\mathrm{Li}$-free gas (diluted with pristine gas) coming from fast-rotating massive stars that are not able to produce fresh $\mathrm{Li}$ (Decressin et al. 2007). On the other hand, the high $A(\mathrm{Li})$ measured among the $2 \mathrm{P}-\mathrm{Li}$ normal stars suggests a production of new Li. Massive AGB stars are potentially able to produce Li through the Cameron-Fowler mechanism and they may explain the Li abundance in most of the Na-rich stars.

However, theoretical models for $A(\mathrm{Li})$ in GCs based on AGB stars as main polluters depend on several parameters, in particular the yields of the AGB models and the lithium abundance of the pristine material; the production of Li in AGB stars is sensitive to the stellar mass (see D'Antona et al. 2012, for a discussion on these assumptions). D'Antona et al. (2012) discussed for M4 the case of polluters ejecta that are Li free (a case similar to that of fast-rotating massive stars) and diluted with pristine gas, finding only a mildly lower (by about $0.1 \mathrm{dex}) A(\mathrm{Li})$ in $2 \mathrm{P}$ stars with respect to $1 \mathrm{P}$ stars. Hence, high $A(\mathrm{Li})$ in Na-rich stars could also be explained with polluter stars not able to produce fresh $\mathrm{Li}$, depending on the details of the dilution process. The need for some fine-tuning to reproduce the uniform Li content observed in stars with a large spread in $\mathrm{Na}$ abundance, as well as uncertainties in the theoretical mass-loss rates and $\mathrm{Li}$ yields, reduces the predictive power of these models.

Another peculiarity of Omega Centauri is the low Li abundance in its metal-rich stars. The stars with $[\mathrm{Fe} / \mathrm{H}]>-1.3 \mathrm{dex}$ exhibit an unexpected depletion of $A(\mathrm{Li})$ that is not observed in GCs with comparable metallicities (i.e. M4 and NGC 2808), which show in most of their (dwarf or LRGB) stars $A(\mathrm{Li})$ similar to that measured in metal-poor clusters. We demonstrated that the effect of high metallicity, high He content, and/or young age (that could characterize the high-metallicity stars in Omega Centauri) cannot explain the observed values, under the assumption that all the stars of Omega Centauri formed with the same initial $A(\mathrm{Li})$. Hence, we conclude that the MR-Li-depleted stars formed from a gas already depleted in $A(\mathrm{Li})$ (similar to the $2 P$ $\mathrm{Li}$-depleted stars) and the observed Li depletion is not an effect of the FDU. According to this finding, we should observe in the metal-rich main sequence stars of Omega Centauri a surface $A(\mathrm{Li})$ smaller than the typical value of the Spite Plateau. The observation of these stars is very challenging owing to their faintness. Monaco et al. (2010) and Pancino et al. (2011b) provided upper limits for $A(\mathrm{Li})$ in 18 stars belonging to the metal-rich subgiant branch of Omega Centauri. Unfortunately, the upper limits for the initial Li content of these stars do not provide conclusive answers.

An additional hurdle with respect to mono-metallic GCs is the possible age spread of the stellar populations of Omega Centauri. There is no general consensus about the relative ages of the different stellar components of the system, in particular between the main (metal-poor) population and the most metal-rich population, which could be virtually coeval (see e.g. Tailo et al. 2016) or several Gyr younger (see e.g. Villanova et al. 2014). If the metallicity enrichment of Omega Centauri took place on short timescales, and in particular if the metal-rich stars are coeval or only slightly younger than the other populations, the most metal-rich stars have formed before other sources of $\mathrm{Li}$ production occur, such as novae and cosmic rays spallation (see e.g. Romano et al. 1999).

Detailed theoretical models for the $\mathrm{Li}-\mathrm{Na}$ behaviour in Omega Centauri are not available so far. In particular, future theoretical models for Omega Centauri need to simultaneously explain: (i) the existence of stars with similar $A(\mathrm{Li})$ but different
[Na/Fe] (1P and 2P-Li-normal); (ii) the existence of stars with similar $[\mathrm{Na} / \mathrm{Fe}]$ but different $A(\mathrm{Li})(2 \mathrm{P}-\mathrm{LiR}$ and $2 P$-Li-depleted); and (iii) the $M R$-Li-depleted component with the lowest $A(\mathrm{Li})$ and the highest $[\mathrm{Na} / \mathrm{Fe}]$. The discussed set of abundances will be crucial to put new constraints on the chemical enrichment history of this stellar system.

Acknowledgements. The authors thank the anonymous referee for his/her useful comments and suggestions. SV gratefully acknowledges the support provided by Fondecyt reg. n. 1170518. LM acknowledges the support from "Proyecto interno" of the Universidad Andres Bello. AM warmly thanks Federico Montaguti and Olma for their essential support during the last year.

\section{References}

Alonso, A., Arribas, S., \& Martinez-Roger, C. 1998, A\&AS, 131, 209 Alonso, A., Arribas, S., \& Martinez-Roger, C. 1999, A\&AS, 140, 261 Bastian, N., \& Lardo, C. 2015, MNRAS, 453, 357

Bastian, N., \& Lardo, C. 2018, ARA\&A, 56, 83

Bedin, L. R., Piotto, G., Anderson, J., et al. 2004, ApJ, 605, L125

Bekki, K., \& Freeman, K. C. 2003, MNRAS, 346, L11

Bellazzini, M., Ferraro, F. R., Sollima, A., Pancino, E., \& Origlia, L. 2004, A\&A, 424, 199

Bellini, A., Piott, G., Bedin, L. R., et al. 2009, A\&A, 493, 958

Bessell, M. S. 1979, PASP, 91, 589

Bonifacio, P. 2002, A\&A, 390, 91

Cayrel, R. 1988, in The Impact of Very High S/N Spectroscopy on Stellar Physics, eds. G. Cayrel de Strobel, \& M. Spite, IAU Symp., 132, 345

Carpenter, J. M. 2001, AJ, 121, 2851

Carretta, E., Bragaglia, A., Gratton, R., et al. 2009a, A\&A, 505, 117

Carretta, E., Bragaglia, A., Gratton, R., \& Lucatello, S. 2009b, A\&A, 505, 139

Carretta, E., Bragaglia, A., Gratton, R., D’Orazi, V., \& Lucatello, S. 2009c, A\&A, 508, 695

D'Antona, F., D'Ercole, A., Carini, R., Vesperini, E., \& Ventura, P. 2012, MNRAS, 426, 1710

Decressin, T., Charbonnel, C., \& Meynet, G. 2007, A\&A, 475, 859

Dekker, H., D’Odorico, S., Kaufer, A., Delabre, B., \& Kotzlowski, H. 2000, SPIE, 4008, 534

D’Ercole, A., Vesperini, E., D’Antona, F., McMillan, S. L. W., \& Recchi, S. 2008, MNRAS, 391, 825

D’Ercole, A., D’Antona, F., Ventura, P., Vesperini, E., \& McMillan, S. L. W. 2010, MNRAS, 407, 854

Dobrovolskas, V., Kučinskas, A., Bonifacio, P., et al. 2014, A\&A, 565, A121

D'Orazi, V., Lucatello, S., Gratton, R., et al. 2010, ApJ, 713, L1

D'Orazi, V., Angelou, G. C., Gratton, R. G., et al. 2014, ApJ, 791, 39

D'Orazi, V., Gratton, R. G., Angelou, G. C., et al. 2015, MNRAS, 449, 4038

Ferraro, F. R., Sollima, A., Pancino, E., et al. 2004, ApJ, 603, L81

Freyhammer, L. M., Monelli, M., Bono, G., et al. 2005, ApJ, 623, 860

Gonzalez Hernandez, J. I., Bonifacio, P., Caffau, E., et al. 2009, A\&A, 505, L13

Gratton, R. G., Carretta, E., \& Bragaglia, A. 2012, A\&ARv, 20, 50

Harris, W. E. 1996, AJ, 112, 1487

Johnson, C. O., Pilachowski, C. A., Simmerer, J., \& Schwenk, D. 2008, ApJ, 681,1505

Johnson, C. O., Pilachowski, C. A., Michael, R. R., \& Fulbright, J. P. 2009, ApJ, 698, 2048

Johnson, C. I., \& Pilachowski, C. A. 2010, ApJ, 722, 1373

Kraft, R. P., Sneden, C., Langer, G. E., \& Prosser, C. F. 1992, AJ, 104, 645

Kurucz, R. L. 2005, Mem. Soc. Astron. It. Suppl., 8, 14

Laird, J. B., Rupen, M. P., Carney, B. W., \& Latham, D. W. 1988, AJ, 96, 1908

Lee, Y.-W., Joo, J.-M., Sohn, Y.-J., et al. 1999, Nature, 402, 55

Lind, K., Asplund, M., Barklem, P. S., \& Belyaev, A. K. 2008, A\&A, 528, 103

Lind, K., Primas, F., Charbonnel, C., Grundahl, F., \& Asplund, M. 2009, A\&A, 503,545

Lind, K., Asplund, M., Barklem, P. S., \& Belyaev, A. K. 2011, A\&A, 528, A102

Marino, A. F., Milone, A. P., Piotto, G., et al. 2011, ApJ, 731, 64

McCall, M. L. 2004, AJ, 128, 2144

Meynet, G., Ekström, S., \& Maeder, A. 2006, A\&A, 447, 623

Meszaros, S., Martell, S. L., Shetrone, M., et al. 2015, AJ, 149, 153

Milone, A. P., Bedin, L. R., Piotto, G., et al. 2008, ApJ, 673, 241

Monaco, L., Bonifacio, P., Sbordone, L., Villanova, S., \& Pancino, E. 2010, A\&A, 519, L3

Monaco, L., Villanova, S., Bonifacio, P., et al. 2012, A\&A, 539, A157

Mucciarelli, A. 2013, ArXiv e-prints [arXiv:1311.1403]

Mucciarelli, A., Salaris, M., Lovisi, L., et al. 2011, MNRAS, 412, 81 
A\&A 618, A134 (2018)

Mucciarelli, A., Salaris, M., \& Bonifacio, P. 2012, MNRAS, 419, 2195

Mucciarelli, A., Pancino, E., Lovisi, L., Ferraro, F. R., \& Lapenna, E. 2013, ApJ, 766,78

Mucciarelli, A., Salaris, M., Bonifacio, P., Monaco, L., \& Villanova, S. 2014, MNRAS, 444, 1812

Norris, J. E. 2004, ApJ, 612, L25

Pancino, E., Ferraro, F. R., Bellazzini, M., Piotto, G., \& Zoccali, M. 2000, ApJ, 534, L83

Pancino, E., Mucciarelli, A., Sbordone, L., et al. 2011a, A\&A, 527, A18

Pancino, E., Mucciarelli, A., Bonifacio, P., Monaco, L., \& Sbordone, L. 2011b, A\&A, 534, A53

Pancino, E., Romano, D., Tang, B., et al. 2017, A\&A, 601, A112

Pasquini, L., Avila, G., Allaert, E., et al. 2000, SPIE, 4008, 129

Pasquini, L., Bonifacio, P., Molaro, P., et al. 2005, A\&A, 441, 549

Pietrinferni, A., Cassisi, S., Salaris, M., \& Castelli, F. 2006, ApJ, 642, 797

Piotto, G., Villanova, S., Bedin, L. R., et al. 2005, ApJ, 621, 777

Prantzos, N., \& Charbonnel, C. 2006, A\&A, 458, 135

Renzini, A. 2008, MNRAS, 391, 354
Renzini, A., D'Antona, F., Cassisi, S., et al. 2015, MNRAS, 454, 4197

Romano, D., Matteuci, F., Molaro, P., \& Bonifacio, P. 1999, A\&A, 352, 117

Sbordone, L., Bonifacio, P., Castelli, F., \& Kurucz, R. L. 2004, MSAIS, 5 , 93

Shen, Z.-X., Bonifacio, P., Pasquini, L., \& Zaggia, S. 2010, A\&A, 524, L2

Skrutskie, M. F., Cutri, R. M., Stiening, R., et al. 2006, AJ, 131, 1163

Smith, G. H., \& Norris, J. 1982, ApJ, 254, 149

Sollima, A., Ferraro, F. R., Pancino, E., \& Bellazzini, M. 2005a, MNRAS, 357, 265

Sollima, A., Pancino, E., Ferraro, F. R., et al. 2005b, ApJ, 634, 332

Sollima, A., Ferraro, F. R., Bellazzini, M., et al. 2007, ApJ, 654, 915

Stetson, P. B., \& Pancino, E. 2008, PASP, 120, 1332

Tailo, M., Di Criscienzo, M., D’Antona, F., Caloi, V., \& Ventura, P. 2016, MNRAS, 457, 4525

Ventura, P., D’Antona, F., Mazzitelli, I., \& Gratton, R. G. 2001, ApJ, 550, L65

Villanova, S., Geisler, D., Gratton, R. G., \& Cassisi, S. 2014, ApJ, 791, 107 Willman, B., \& Strader, J. 2012, AJ, 144, 76 